\title{
Atlas of low-mass young stellar object disks from mid-infrared interferometry
}

\author{
J. Varga ${ }^{1}$, P. Ábrahám ${ }^{1}$, Th. Ratzka ${ }^{2}$, J. Menu ${ }^{3,5}$, K. Gabányi ${ }^{1,4}$, Á.
} Kóspál $^{1,5}$, R. van Boekel ${ }^{5}$, L. Mosoni ${ }^{1}$, Th. Henning ${ }^{5}$

${ }^{1}$ Konkoly Observatory, Budapest, Hungary (email: varga.jozsef@csfk.mta.hu)

${ }^{2}$ Institute for Physics/IGAM, University of Graz, Austria

${ }^{3}$ Instituut voor Sterrenkunde, KU Leuven, Belgium

${ }^{4}$ FÖMI Satellite Geodetic Observatory, Budapest, Hungary

${ }^{5}$ MPIA Heidelberg, Germany

\begin{abstract}
We present our approach of visibility modeling of disks around low-mass $\left(<2 M_{\odot}\right)$ young stellar objects (YSOs). We compiled an atlas based on mid-infrared interferometric observations from the MIDI instrument at the VLTI. We use three different models to fit the data. These models allow us to determine overall sizes (and the extent of the inner gaps) of the modeled circumstellar disks.
\end{abstract}

Keywords. planetary systems: protoplanetary disks, stars: pre-main-sequence,techniques: interferometric, atlases, accretion disks

\section{Introduction}

Long-baseline interferometry offers the possibility to achieve the angular resolution required to resolve the planet-forming disks of young stellar objects (YSOs). Observations with the Midinfrared Interferometric Instrument (MIDI, Leinert et al. 2003) at the Very Large Telescope Interferometer (VLTI) provide a wealth of information about the structure of the protoplanetary disks and the spatial distribution of the dust species therein.

We are working on an atlas for low-mass YSOs with all data reduced in a homogeneous manner, and determine YSO disk sizes and morphologies via model fitting. Our goal is to analyze the distribution of disk sizes and discuss different evolutionary scenarios among the low-mass YSO population similarly to Menu et al. (2015), who investigated intermediate-mass young stars. This work supports the preparation of science observations with MATISSE, the successor of MIDI at the VLTI. Our sample contains 85 sources, of which 44 are T Tauri stars, 24 are Herbig Ae stars, and 13 are stars with debris disks. We include Herbig stars in our sample only if they have $T_{\text {eff }}<10000 \mathrm{~K}$. The resulting small overlap with the sample of Menu et al. (2015) allows to validate our models. Data reduction was done in the same way as in Menu et al. (2015).

\section{Visibility modeling}

In Fig. 1 we plot correlated fluxes measured at $10.7 \mu \mathrm{m}$ as a function of projected baseline length. To fit the data points we used the temperature gradient model of Menu et al.(2015):

$$
I_{\nu}=\tau_{\nu} B_{\nu}(T(R)), \text { with } T(R)=T_{\text {sub }}\left(\frac{R}{R_{\text {sub }}}\right)^{-q}
$$

Here $T_{\text {sub }}$ is the dust sublimation temperature, fixed at $1500 \mathrm{~K} . R_{\text {sub }}$ is the sublimation radius, calculated from the known luminosity $(L)$ of the central star. The disk inclination is not fitted, we assume face-on disks.

- Model 1. Disk model with fixed inner and outer radii from Menu et al. (2015). The radial range of the model is $R_{\mathrm{sub}}<R<R_{\text {out }}=300$ au. The fitted parameters are the temperature 

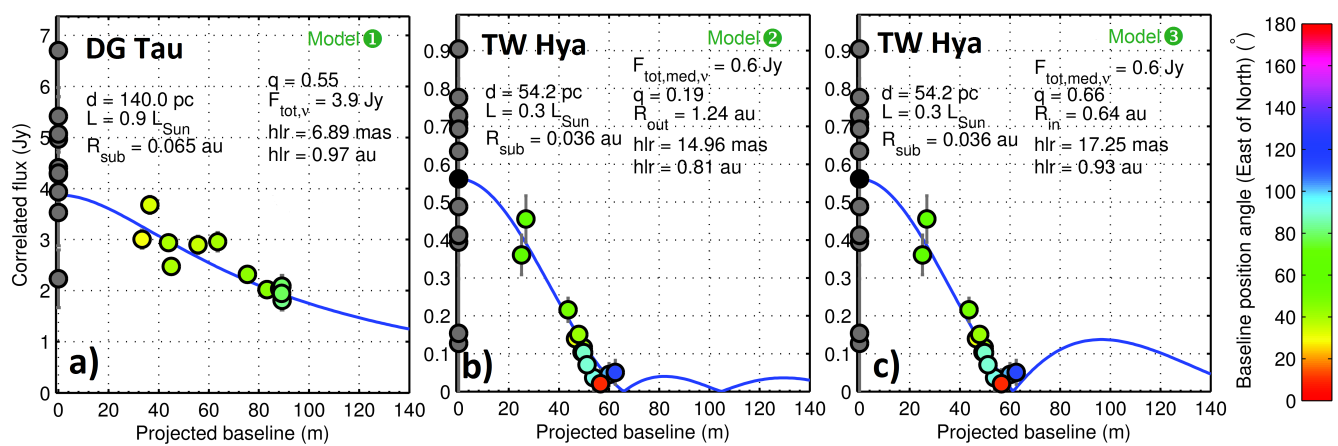

Figure 1. Correlated fluxes as a function of projected baseline length for DG Tau and TW Hya, showing the results of our model fitting (blue lines). The color of the symbols represents the baseline position angles. See the text for more details.

power-law exponent $q$, and the total flux $F_{\text {tot, }, \nu}$. From $q$ a half-light radius $(h l r)$ is calculated. DG Tau was fitted with this model in panel a).

- Model 2. A ring model with variable outer radius. Here $F_{\text {tot }, \nu}$ is not fitted, but calculated as the median of all the measured total fluxes. The inner radius is fixed at $R_{\mathrm{sub}}$, but the outer radius $R_{\text {out }}$ is a free parameter. We again fit $q$. See panel b) for an example (TW Hya).

- Model 3. Disk model with inner gap. Again, $F_{\mathrm{tot}, \nu}$ is the median of the total fluxes. The outer radius is fixed at $300 \mathrm{au}$, but the inner radius $R_{\mathrm{in}}$ is a fitted parameter. Thus, this model can account for inner disk clearing. Again, $q$ is a free parameter. See panel c) for an example (TW Hya).

Models 2 and 3 fit well the observed data of TW Hya, which has a known transition disk with an inner cavity (Calvet et al. 2002).. We found $R_{\mathrm{in}}=0.64 \mathrm{au}$, which is in good agreement with previous results ( $0.5 \mathrm{au}$ ) (Ratzka et al. 2007; Menu et al. 2014). Both models work well despite the different geometries. This is caused by two factors: a) the bulk of the radiation comes from a narrow ring, b) the lack of data points for baselines $>60 \mathrm{~m}$ prevents us from constraining more accurately the disk structure.

\section{Acknowledgements}

This work was supported by the Hungarian Research Fund OTKA grant K101393 and by the Momentum grant of the MTA CSFK Lendület Disk Research Group. The authors are thankful for the support of the Fizeau Exchange Visitor Program (OPTICON/FP7).

\section{References}

Calvet, N., D'Alessio, P., Hartmann, L., et al. 2002, ApJ, 568, 1008

Jaffe, W. J. 2004, Proceedings of the SPIE, 5491, 715

Leinert, C., Graser, U., Przygodda, F., et al. 2003, ApESSS, 286, 73

Menu, J., van Boekel, R., Henning, T., et al. 2014, A\&A, 564, A93

Menu, J., van Boekel, R., Henning, T., et al. 2015, A\&A A, 581, A107

Ratzka, T., Leinert, C., Henning, T., et al. 2007, A\&SA, 471, 173 